\title{
The mass of the Milky Way: Limits from a newly assembled set of halo objects ${ }^{\star}$
}

\author{
T. Sakamoto ${ }^{1}$, M. Chiba ${ }^{2}$, and T. C. Beers ${ }^{3}$ \\ ${ }^{1}$ Department of Astronomical Science, The Graduate University for Advanced Studies, Mitaka, Tokyo 181-8588, Japan \\ 2 National Astronomical Observatory, Mitaka, Tokyo 181-8588, Japan \\ ${ }^{3}$ Department of Physics \& Astronomy, Michigan State University, East Lansing, MI 48824, USA
}

Received 7 August 2002 / Accepted 14 October 2002

\begin{abstract}
We set new limits on the mass of the Milky Way, making use of the latest kinematic information for Galactic satellites and halo objects. Our sample consists of 11 satellite galaxies, 137 globular clusters, and 413 field horizontal-branch (FHB) stars up to distances of $10 \mathrm{kpc}$ from the Sun. Roughly half of the objects in this sample have measured proper motions, permitting the use of their full space motions in our analysis. In order to bind these sample objects to the Galaxy, their rest-frame velocities must be lower than their escape velocities at their estimated distances. This constraint enables us to show that the mass estimate of the Galaxy is largely affected by several high-velocity objects (Leo I, Pal 3, Draco, and a few FHB stars), not by a single object alone (such as Leo I), as has often been the case in past analyses. We also find that a gravitational potential that gives rise to a declining rotation curve is insufficient to bind many of our sample objects to the Galaxy; a possible lower limit on the mass of the Galaxy is about $2.2 \times 10^{12} M_{\odot}$. To be more quantitative, we adopt a Bayesian likelihood approach to reproduce the observed distribution of the current positions and motions of the sample, in a prescribed Galactic potential that yields a flat rotation curve. This method enables a search for the most likely total mass of the Galaxy, without undue influence in the final result arising from the presence or absence of Leo I, provided that both radial velocities and proper motions are used. Although the best mass estimate depends somewhat on the model assumptions, such as the unknown prior probabilities for the model parameters, the resultant systematic change in the mass estimate is confined to a relatively narrow range of a few times $10^{11} M_{\odot}$, owing to our consideration of many FHB stars. The most likely total mass derived from this method is $2.5_{-1.0}^{+0.5} \times 10^{12} M_{\odot}$ (including Leo I), and $1.8_{-0.7}^{+0.4} \times 10^{12} M_{\odot}$ (excluding Leo I). The derived mass estimate of the Galaxy within the distance to the Large Magellanic Cloud $(\sim 50 \mathrm{kpc})$ is essentially independent of the model parameters, yielding $5.5_{-0.2}^{+0.0} \times 10^{11} M_{\odot}$ (including Leo I) and $5.4_{-0.4}^{+0.1} \times 10^{11} M_{\odot}$ (excluding Leo I). Implications for the origin of halo microlensing events (e.g., the possibility of brown dwarfs as the origin of the microlensing events toward the LMC, may be excluded by our lower mass limit) and prospects for more accurate estimates of the total mass are also discussed.
\end{abstract}

Key words. Galaxy: halo - Galaxy: fundamental parameters - Galaxy: kinematics and dynamics - stars: horizontal-branch

\section{Introduction}

Over the past decades, various lines of evidence have revealed that the mass density in the Milky Way is largely dominated by unseen dark matter, from the solar neighborhood to the outer reaches of the halo (e.g., Fich \& Tremaine 1991). Moreover, the presence of a dark component similar to that found in our own Galaxy appears to be a generic feature in external galaxies, as inferred from, e.g., flat rotation curves in their outer parts, the presence of (a gravitationally bound) hot plasma in early-type galaxies, and the observed gravitational lensing of background sources (e.g., Binney \& Tremaine 1987). A determination of

Send offprint requests to: T. Sakamoto,

e-mail: sakamoto@pluto.mtk.nao.ac.jp

* Table 1 is only available at the CDS via anonymous ftp to cdsarc.u.strasbg.fr (130.79.128.5) or via

http://cdsweb.u-strasbg.fr/cgi-bin/qcat?J/A+A/397/899 the extent over which such dark-matter-dominated mass distributions apply for most galaxies, including our own, is of great importance for understanding the role of dark matter in galaxy formation and dynamical evolution. In particular, the mass estimate of the Galaxy is closely relevant to understanding the origin of the microlensing events toward the Large Magellanic Cloud (LMC) (e.g., Alcock et al. 2000; Alcock et al. 2001).

While mass estimates of external galaxies can, in principle, be obtained in a straightforward fashion using various dynamical probes, the total mass of the Galaxy remains rather uncertain, primarily due to the lack of accurate observational information for tracers located in its outer regions, where the dark matter dominates. The precise shape of the outer rotation curve, as deduced from H II regions and/or H I gas clouds (e.g., Honma \& Sofue 1997), is still uncertain because its determination requires knowledge of accurate distances to these tracers (Fich \& Tremaine 1991). Also, interstellar gas can be traced 
only up to $\sim 20 \mathrm{kpc}$ from the Galactic Center, and hence provides no information concerning the large amount of dark matter beyond this distance.

The most suitable tracers for determination of the mass distribution in the outer halo of the Galaxy are the distant luminous objects, such as satellite galaxies, globular clusters, and halo stars on orbits that explore its farthest reaches (e.g., Miyamoto et al. 1980; Little \& Tremaine 1987; Zaritsky et al. 1989; Kochanek 1996; Wilkinson \& Evans 1999, hereafter WE99). However, the limited amount of data presently available on the full space motions of these tracers, and the small size of the available samples, have stymied their use for an accurate determination of the Galaxy's mass. In particular, most previous mass estimates (except for WE99, see below) depend quite sensitively on whether or not a distant satellite, Leo I, is bound to the Galaxy. Leo I has one of the largest radial velocities of the known satellites, despite its being the second most distant satellite from the Galaxy (Mateo 1998; Held et al. 2001). As a consequence, estimates of the total mass of the Galaxy are much more uncertain (by as much as an order of magnitude) than, for instance, the value of the circular speed in the solar neighborhood (Kerr \& Lynden-Bell 1986; Fich \& Tremaine 1991; Miyamoto \& Zhu 1998; Méndez et al. 1999).

Recently, by making use of both the observed radial velocities and proper motions of six distant objects, WE99 demonstrated that the use of full space motions can provide a reliable mass estimate of the Galaxy without being largely affected by the presence or absence of Leo I. They also argued that the primary uncertainties in their mass estimate arose from the small size of the data set and the measurement errors in the full space motions, especially the proper motions. This work motivated us to investigate a much larger data set, with more accurate kinematic information, to set tighter limits on the mass of the Galaxy. Specifically, as we show below, there are two objects among the WE99 sample (Draco and Pal 3) that have relatively large velocity errors, yet still play crucial roles in a determination of the Galaxy's mass, so the addition of more (and better) data is important.

Over the past few years, the number of distant satellite galaxies and globular clusters with available proper motions has gradually increased (e.g., Mateo 1998; Dinescu et al. 1999; Dinescu et al. 2000; Dinescu et al. 2001). In addition, another tracer population that is suitable for exploring mass estimates of the Galaxy has become available from the extensive compilation of A-type metal-poor stars by Wilhelm et al. (1999b), which provided radial velocity measurements, as well as estimates of the physical parameters of these stars (e.g., $\left.[\mathrm{Fe} / \mathrm{H}], T_{\text {eff }}, \log g\right)$. Among the Wilhelm et al. sample, the luminous FHB stars are the most useful mass tracers, both because of their intrinsic brightness, and the fact that accurate distance determinations can be inferred from their absolute magnitudes on the horizontal branch (e.g., Carretta et al. 2000). Moreover, there exist proper-motion measurements for many of these stars, provided by both ground- and space-based propermotion catalogs (Klemola et al. 1994; Röser 1996; Platais et al. 1998; Hog et al. 2000), from which full space motions may be derived.
In this paper we re-visit the mass determination of the Galaxy, based on a sample of 11 satellite galaxies, 137 globular clusters, and 413 FHB stars, out of which 5 satellite galaxies, 41 globular clusters, and 211 FHB stars have measured proper motions. First, we investigate the lowest possible mass that the Galaxy may have, adopting the requirement that the rest-frame velocities of observed sample objects be less than their escape velocities at their present distance from the Galactic center (e.g., Miyamoto et al. 1980; Carney et al. 1988). Secondly, the most likely mass of the Galaxy is calculated, based on a Bayesian likelihood analysis that seeks to reproduce both the current positions and velocities of the sample objects (e.g., Little \& Tremaine 1987; Kochanek 1996; WE99). Because our sample of tracers is, by far, the largest and most accurate one presently available, it is possible to place more reliable limits on the total mass of the Galaxy. In Sect. 2 we describe our sample objects and the assembly of their kinematic data. In Sect. 3 we discuss the influence of the adopted membership of our sample on the mass determination. In Sect. 4 we obtain the most likely total mass of the Galaxy based on a Bayesian likelihood analysis. In Sect. 5 we discuss implications for the origin of the halo microlensing events toward the LMC and the mass estimate of the Local Group, and consider the prospects for more obtaining more accurate estimates of the total mass of the Galaxy in the near future.

\section{Data}

We consider a sample of objects that serve as tracers of the Galactic mass distribution consisting of 11 satellite galaxies, 137 globular clusters, and 413 FHB stars. In the case of the satellite galaxies, all of the basic information for our kinematic analysis, i.e., positions, heliocentric distances, and heliocentric radial velocities, are taken from the compilation of Mateo (1998). For the globular clusters, we adopt the information provided by Harris (1996), including their positions and heliocentric radial velocities, their metal abundances, $[\mathrm{Fe} / \mathrm{H}]$, and the apparent magnitude of the clusters' horizontal branch (HB). The catalog of Wilhelm et al. (1999b) is our source of similar information for the FHB stars. We obtain an internally consistent set of distance estimates for the globular clusters and the FHB stars from the recently derived relationship between the absolute magnitude of the $\mathrm{HB}, M_{V}(\mathrm{HB})$, and $[\mathrm{Fe} / \mathrm{H}]$, by Carretta et al. (2000),

$M_{V}(\mathrm{HB})=(0.18 \pm 0.09)([\mathrm{Fe} / \mathrm{H}]+1.5)+(0.63 \pm 0.07)$.

Clearly, we have assumed that there is no large offset between the absolute magnitudes of FHB stars and their counterpart HB stars in the globular clusters (a view also supported by the recent work of Carretta et al. 2000). Figure 1 shows the spatial distribution of the globular clusters, satellite galaxies, and FHB stars on the plane perpendicular to the Galactic disk, where $X$ axis connects the Galactic center $(X=0)$ and the $\operatorname{Sun}(X=8.0 \mathrm{kpc})$. The filled and open symbols denote the objects with and without proper-motion measurements, respectively. Satellite galaxies are the most distant tracers, with Galactocentric distances, $r$, greater than $50 \mathrm{kpc}$. The globular clusters extend out to almost $r=40 \mathrm{kpc}$, while the present 
(a)

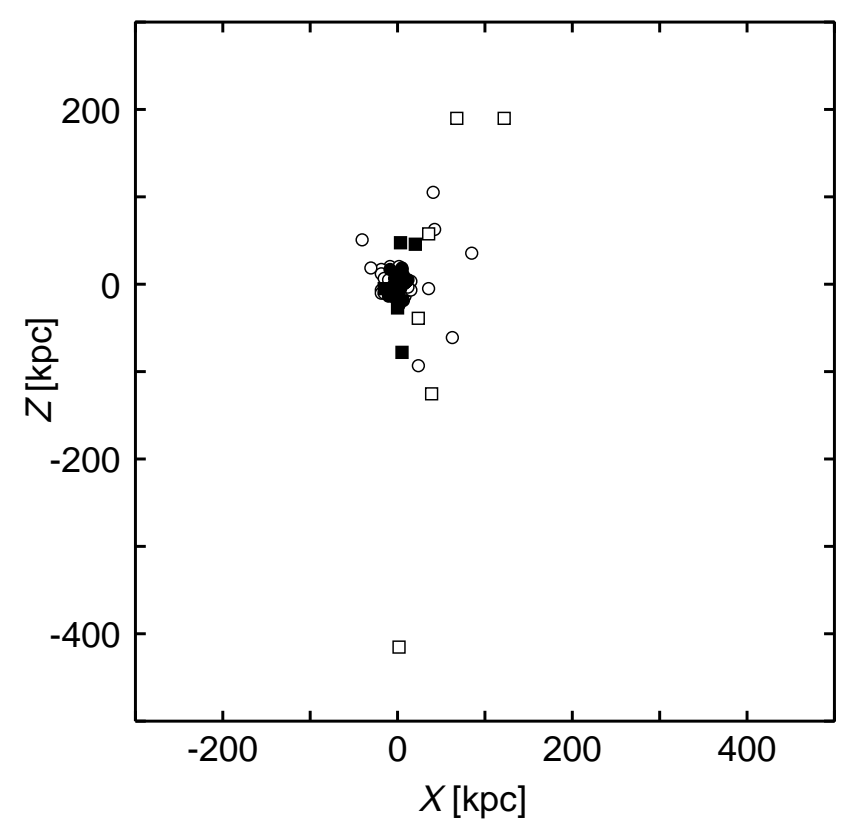

(b)

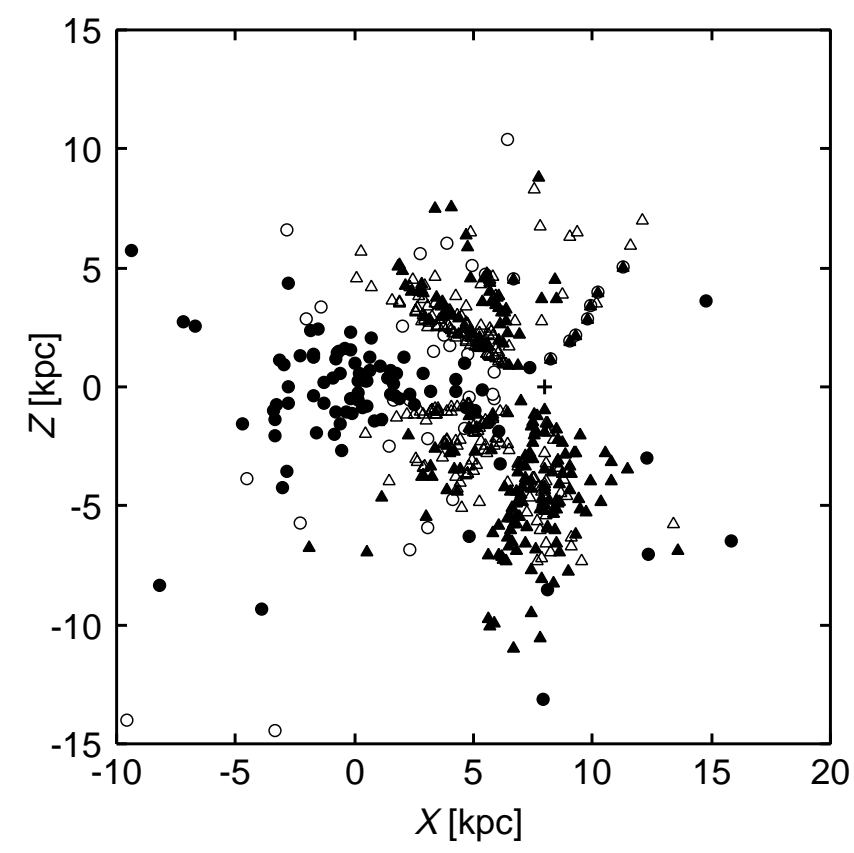

Fig. 1. Spatial distributions of satellite galaxies (squares), globular clusters (circles), and FHB stars (triangles) on the plane perpendicular to the Galactic disk, where the $X$ axis connects the Galactic center $(X=0)$ and the Sun $(X=8.0 \mathrm{kpc})$. The filled and open symbols denote the objects with and without available proper motions, respectively. The plus sign in panel b) denotes the position of the Sun, $(X, Y)=(8.0,0)$

sample of FHB stars are confined to locations within $10 \mathrm{kpc}$ of the Sun. Thus, our sample objects are widely, though not uniformly, distributed throughout the volume of the Galaxy.

Among these sample objects there exist proper-motion measurements for 5 of the satellite galaxies, 41 of the globular clusters, and 211 of the FHB stars. The proper motion data for the LMC, Sculptor, and Ursa Minor are taken from WE99, whereas those for Sagittarius and Draco are taken from Irwin et al. (1996) and Scholz \& Irwin (1994), respectively. The proper motions for most of the globular clusters have been compiled by Dinescu et al. (1999). We adopt the data from this source, except for two globular clusters with recently revised proper-motion measurements (NGC 6254: Chen et al. 2000; NGC 4147: Wang et al. 2000), and for three additional globular clusters compiled recently (Pal 13: Siegel et al. 2000; Pal 12: Dinescu et al. 2000; NGC 7006: Dinescu et al. 2001). Proper motions for 211 of the FHB stars in the Wilhelm et al. (1999b) sample are available from one or more existing proper-motion catalogs. These include the STARNET Catalog (Röser 1996), the Yale-San Juan Southern Proper Motion Catalog (SPM 2.0: Platais et al. 1998), the Lick Northern Proper Motion Catalog (NPM1: Klemola et al. 1994), and the TYCHO-2 Catalog (Hog et al. 2000). Many of these FHB stars have been independently measured in two or more catalogs, so that by combining all measurements one can reduce the statistical errors, as well as minimize any small remaining systematic errors in the individual catalogs, as was argued in Martin \& Morrison (1998) and Beers et al. (2000).

We estimate average proper motions, $\langle\mu\rangle$, and their errors, $\left\langle\sigma_{\mu}\right\rangle$, weighted by the inverse variances, as

$$
\begin{aligned}
<\mu> & =\left(\sum_{i=1}^{n} \mu_{i} / \sigma_{\mu_{i}}^{2}\right) /\left(\sum_{i=1}^{n} 1 / \sigma_{\mu_{i}}^{2}\right) \\
<\sigma_{\mu}> & =\left(\sum_{i=1}^{n} 1 / \sigma_{\mu_{i}}^{2}\right)^{-1 / 2},
\end{aligned}
$$

where $n$ denotes the number of measurements. Table 1 (available at the CDS) lists these compilations, as well as the estimated distances to the FHB stars, where $r$ and $R V$ denote the Galactocentric distances and heliocentric radial velocities, respectively. Typical errors in the reported proper-motion mea-

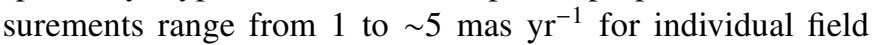
stars, whereas those for the satellite galaxies and globular clus-

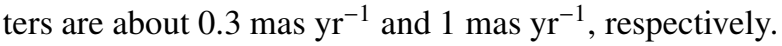

We assume a circular rotation speed for the Galaxy of $V_{\mathrm{LSR}}=220 \mathrm{~km} \mathrm{~s}^{-1}$ at the location of the Sun (i.e. $R_{\odot}=8.0 \mathrm{kpc}$ along the disk plane) and a solar motion of $(U, V, W)=(-9,12$, 7) $\mathrm{km} \mathrm{s}^{-1}$ (Mihalas \& Binney 1981), where $U$ is directed outward from the Galactic Center, $V$ is positive in the direction of Galactic rotation, and $W$ is positive toward the North Galactic Pole $^{1}$. We then calculate the space motions of our tracers, as well as the errors on these motions, fully taking into account the reported measurement errors in the radial velocities of the individual satellite galaxies (typically a few $\mathrm{km} \mathrm{s}^{-1}$ ), adopting a typical radial-velocity error for other objects $\left(10 \mathrm{~km} \mathrm{~s}^{-1}\right)$, the measurement errors assigned to the proper motions of each object (when available, adopting a mean error for the source catalog when not), and distance errors for the satellite

1 Dehnen \& Binney (1998) derived the solar motion of $(U, V, W)=$ $(-10.0,5.3,7.2) \mathrm{km} \mathrm{s}^{-1}$ based on Hipparcos data. Because the difference between this and the currently adopted solar motion is only a few $\mathrm{km} \mathrm{s}^{-1}$, it gives little influence on the mass estimate. 
galaxies (10\% relative to the measured ones), or as obtained from Eq. (1) for the globular clusters and FHB stars.

It is worth noting that the reported proper motions of the FHB stars in our sample may yet contain unknown systematics with respect to their absolute motions in a proper reference frame; this caution applies to the globular clusters and satellite galaxies as well. It is an important goal to make efforts to reduce the systematic, as well as random, errors in the proper motions upon which studies of Galactic structure and kinematic studies are based, using much higher precision astrometric observations than have been obtained to date.

\section{Roles of the sample in the mass limits}

If we model the Galaxy as an isolated, stationary mass distribution, and assume that all of our tracer objects are gravitationally bound to it, then the rest-frame velocities of all objects, $V_{\mathrm{RF}}$, must be less than their escape velocities, $V_{\mathrm{esc}}=\sqrt{2 \psi}$, where $\psi$ denotes the gravitational potential of the Galaxy. A number of previous researchers have adopted this method for estimation of the mass of the Galaxy (e.g., Fricke 1949; Miyamoto et al. 1980; Carney et al. 1988; Leonard \& Tremaine 1990; Dauphole \& Colin 1995). Note that the mass estimate obtained in this fashion is largely dominated by only a small number of highvelocity objects, hence the mass that is derived depends rather sensitively on the selection criteria adopted for such objects. In the present section, instead of deriving an exact mass estimate, we follow this procedure for the purpose of elucidating the role of sample selection in setting limits on the mass of the Galaxy.

To this end we consider two different mass models, in order to investigate the difference in the mass limits obtained by the use of different potentials. Our models, hereafter referred to as Model A and B, are the same as those adopted in WE99 and Johnston et al. (1995) (and also used by Dinescu et al. 1999), respectively.

Model A has spherical symmetry, and results in a flat rotation curve in the inner regions of the Galaxy. The gravitational potential and mass density are given as:

$$
\begin{aligned}
& \psi(r)=\frac{G M}{a} \log \left(\frac{\sqrt{r^{2}+a^{2}}+a}{r}\right), \\
& \rho(r)=\frac{M}{4 \pi} \frac{a^{2}}{r^{2}\left(r^{2}+a^{2}\right)^{3 / 2}},
\end{aligned}
$$

where $a$ is the scale length of the mass distribution, and $M$ is the total mass of the system. The central density of this model is cusped (like $r^{-2}$ ) and falls off as $r^{-5}$ for $r \gg a$. Using Eq. (4), the circular rotation speed is given as $V_{\mathrm{c}}^{2}=G M /\left(r^{2}+a^{2}\right)^{1 / 2}$, so by setting $V_{\mathrm{c}}$ at $r=R_{\odot}$ as $V_{\mathrm{LSR}}=220 \mathrm{~km} \mathrm{~s}^{-1}$ in our standard case, it follows that this model contains one free parameter, $a$, to obtain $M$.

Model B consists of more realistic axisymmetric potentials with three components (the bulge, disk, and dark halo) that reproduce the shape of the Galactic rotation curve (Johnston et al. 1995). The bulge and disk components we adopt are those represented by Hernquist (1990) and Miyamoto \& Nagai (1975) potentials, respectively. All of the parameters included in these potentials are taken from Dinescu et al. (1999)

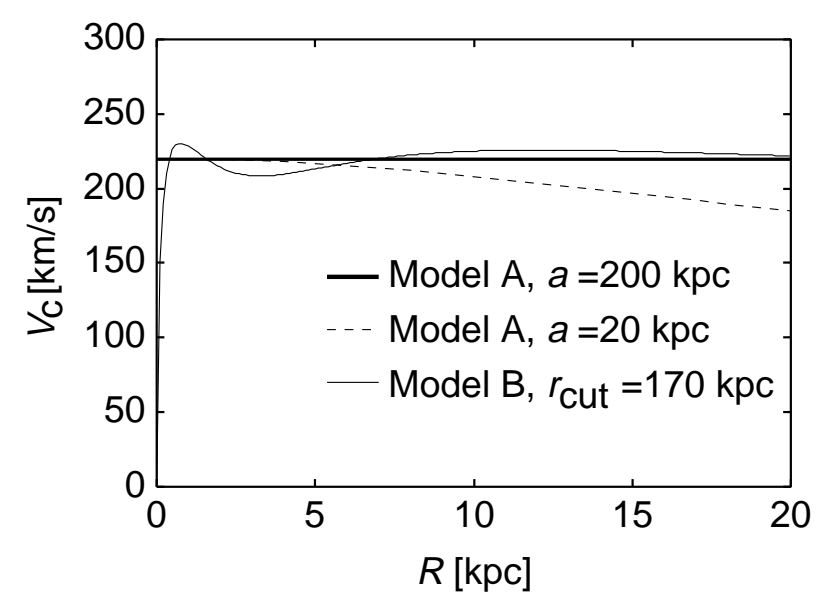

Fig. 2. Rotation curves for Model A and Model B, parameterizations of the mass distributions considered in this paper. See the text for more information on the nature of these models.

(see their Table 3). In order to obtain a finite total mass we assume the following modified logarithmic potential (corresponding to an isothermal-like density distribution) for the dark halo component:

$\psi_{\text {halo }}(r)=\left\{\begin{array}{l}v_{0}^{2} \log \left[1+(r / d)^{2}\right]-\psi_{0}, \quad \text { at } r<r_{\text {cut }} \\ -2 v_{0}^{2} \text { cut } \frac{c}{1+c}, \quad \text { at } r \geq r_{\text {cut }},\end{array}\right.$

$\rho(r)=\frac{2 v_{0}^{2}}{4 \pi G d^{2}} \frac{3+r / d}{(1+r / d)^{3}}$,

where $\psi_{0}$ is defined as

$\psi_{0}=v_{0}^{2}[\log (1+c)+2 c /(1+c)], \quad c=\left(r_{\mathrm{cut}} / d\right)^{2}$,

and we adopt $v_{0}=128 \mathrm{~km} \mathrm{~s}^{-1}$ and $d=12 \mathrm{kpc}$ (Dinescu et al. 1999). This model contains one free parameter, namely the cutoff radius of the dark halo, $r_{\text {cut }}$. Figure 2 shows the rotation curves for $0 \leq R \leq 20 \mathrm{kpc}$, provided by Model A with $a=200 \mathrm{kpc}$ (thick solid line) and Model B with $r_{\text {cut }}=170 \mathrm{kpc}$ (thin solid line), where both curves shown at $R \leq 20 \mathrm{kpc}$ remain unchanged as long as $a, r_{\text {cut }} \gg 20 \mathrm{kpc}$. The circular speed at $R=R_{\odot}$ is $220 \mathrm{~km} \mathrm{~s}^{-1}$ for both mass models. Also shown is the declining rotation curve with increasing radius, as obtained from Model A with $a=20 \mathrm{kpc}$ and $V_{\mathrm{LSR}}=211 \mathrm{~km} \mathrm{~s}^{-1}$ (dashed line).

We plot, in Figs. 3a and 3b, the relationship between the derived escape velocities, $V_{\mathrm{esc}}$, and the rest-frame velocities, $V_{\mathrm{RF}}$, when we adopt Model A with $a=195 \mathrm{kpc}$ and Model B with $r_{\text {cut }}=295 \mathrm{kpc}$, respectively. For the objects without available proper motions (open symbols), we adopt the radial velocities alone as measures of $V_{\mathrm{RF}}$, hence their estimated space velocities are only lower limits. The solid line denotes the boundary between the objects that are bound (below the line) and unbound (above the line) to the Galaxy, respectively, where the positions of the data points relative to the solid line depend on the choice of $a$ or $r_{\text {cut }}$. As these figures clearly indicate, the selection of the smallest value of $a$ or $r_{\text {cut }}$ that places the sample inside the bound region, or equivalently the lower limit on the mass of the Galaxy, is controlled by a few high-velocity objects located near the boundary line at each respective radius 
(a)

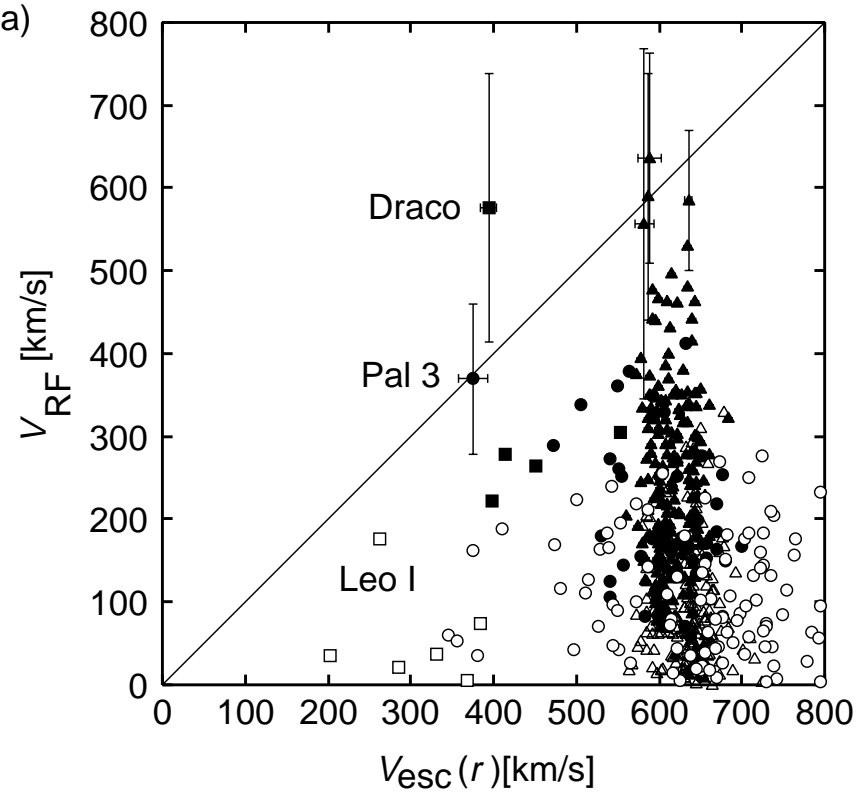

(b)

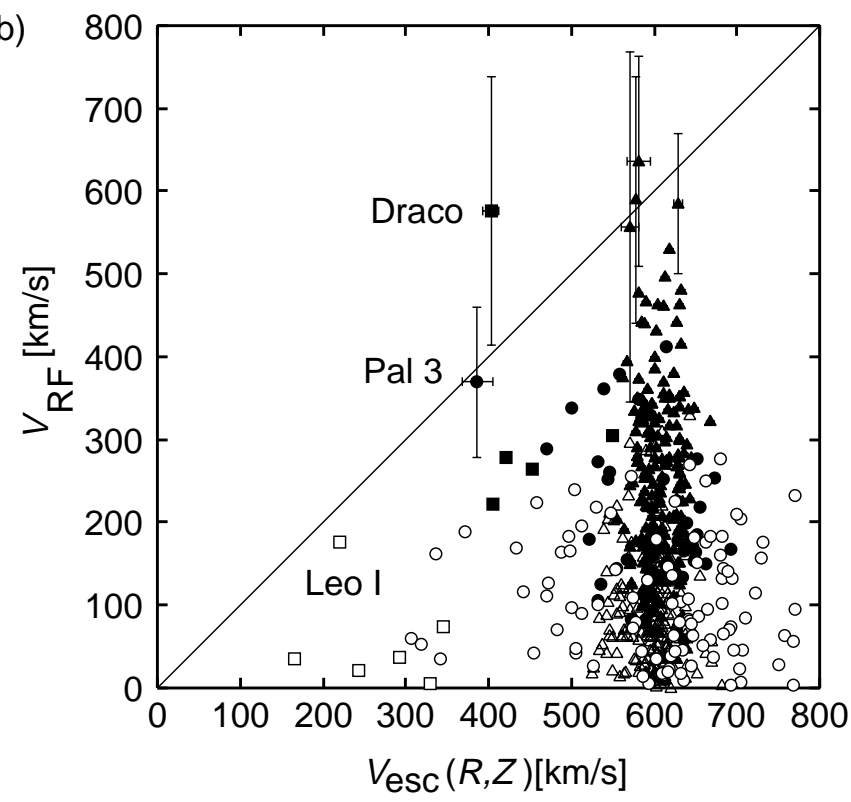

Fig. 3. a) The relation between escape velocities, $V_{\text {esc }}$, and space velocities, $V_{\mathrm{RF}}$, for Model A with $a=195 \mathrm{kpc}$ and $V_{\mathrm{LSR}}=220 \mathrm{~km} \mathrm{~s}^{-1}$. The symbols are the same as those in Fig. 1. The solid line denotes the boundary between the gravitationally bound and unbound objects those in the region below the line are bound to the Galaxy. For the sake of clarity, velocity errors are plotted for only the high-velocity objects relevant to the mass estimate. b) Same as panel a) but for Model B with $r_{\text {cut }}=295 \mathrm{kpc}$.

(or corresponding $\psi$ ). These objects include Leo I (for which only radial velocity information is available), Draco, $\mathrm{Pal} 3$, and several high-velocity FHB stars. This highlights the following important properties of the derived mass limits: (1) If the proper motions of all objects are unavailable, then the mass estimate sensitively depends on the presence or absence of Leo I, as has been noted in previous studies. (2) Compared to case (1), if the available proper motions of the satellite galaxies and globular clusters are taken into account, the constraint provided by Draco and Pal 3 is basically the same as that provided by Leo I. This may explain the result of WE99, which showed that the mass determination is made insensitive to Leo I if the proper motion data of satellite galaxies and globular clusters are taken into account. However, as Fig. 3 indicates, the velocity errors for Draco and Pal 3 are quite large, so these objects place only weak constraints on the mass estimate. (3) If we consider the proper motions of FHB stars, then some FHB stars having high velocities provide the basically the same constraint on the Galaxy's mass as do Leo I, Draco, and Pal 3. These properties suggest that the inclusion of FHB stars with available proper motions is crucial, and that they provide constraints on the mass limit of the Galaxy that depend on neither the inclusion or absence of Leo I nor on the large velocity errors for Draco and Pal 3.

As mentioned above, a determination of the lower mass limit for the Galaxy, using escape velocities, inevitably depends on the selection of a few apparently high-velocity objects from a much larger sample of tracers. While a mass estimate independent of this selection effect will be obtained in Sect. 4, we seek first to obtain a rough measure for the lower mass limit, i.e., the smallest $a$ or $r_{\text {cut }}$ that encloses Pal 3, Draco, and the four highest-velocity FHB stars (for which we mark asterisks after their names in Table 1) inside the bound region, based on a weighted least-squares fitting procedure (weights being inversely proportional to the velocity errors). This exercise yields $a=195_{-85}^{+160} \mathrm{kpc}$ for Model A, and $r_{\text {cut }}=295_{-145}^{+335} \mathrm{kpc}$ for Model B. Using these values, the lower limits to the total mass, $M$, of the Galaxy may be given as $2.2_{-1.0}^{+1.8} \times 10^{12} M_{\odot}$ for Model A and $2.2_{-1.1}^{+2.6} \times 10^{12} M_{\odot}$ for Model B, respectively. Thus, the difference in the derived mass limits is not significant, as long as the rotation curve at outer radii is approximately constant at the adopted value of $220 \mathrm{~km} \mathrm{~s}^{-1}$. It also suggests that the flattened nature of the Model B potential, due to the presence of the disk component, does not affect the results significantly the high-velocity tracers are located at large Galactocentric distances and/or their orbits largely deviate from the disk plane.

In addition to the above experiments, we also consider a mass model that yields a declining rotation curve at outer radii, as was proposed by Honma \& Sofue (1997) from their H I observations. We adopt Model A with $a=20 \mathrm{kpc}$ and $V_{\mathrm{LSR}}=211 \mathrm{~km} \mathrm{~s}^{-1}$ at $R=R_{\odot}$, so as to yield the declining rotation curve at $R>R_{\odot}$ (dashed line in Fig. 2), which is reminiscent of the result in Honma \& Sofue (1997). Figure 4 shows the $V_{\mathrm{RF}}$ vs. $V_{\mathrm{esc}}$ relationship that follows from adoption of this model. As is evident, the total mass obtained from a model that leads to a declining rotation curve is insufficient to bind many of our sample objects to the Galaxy.

\section{Mass determination based on a Bayesian likelihood method}

\subsection{Method}

To obtain a more quantitative measure of the mass of the Galaxy, we examine an alternative method that takes into account all of the positional and kinematic information of the sample objects, in contrast to the use of the high-velocity 


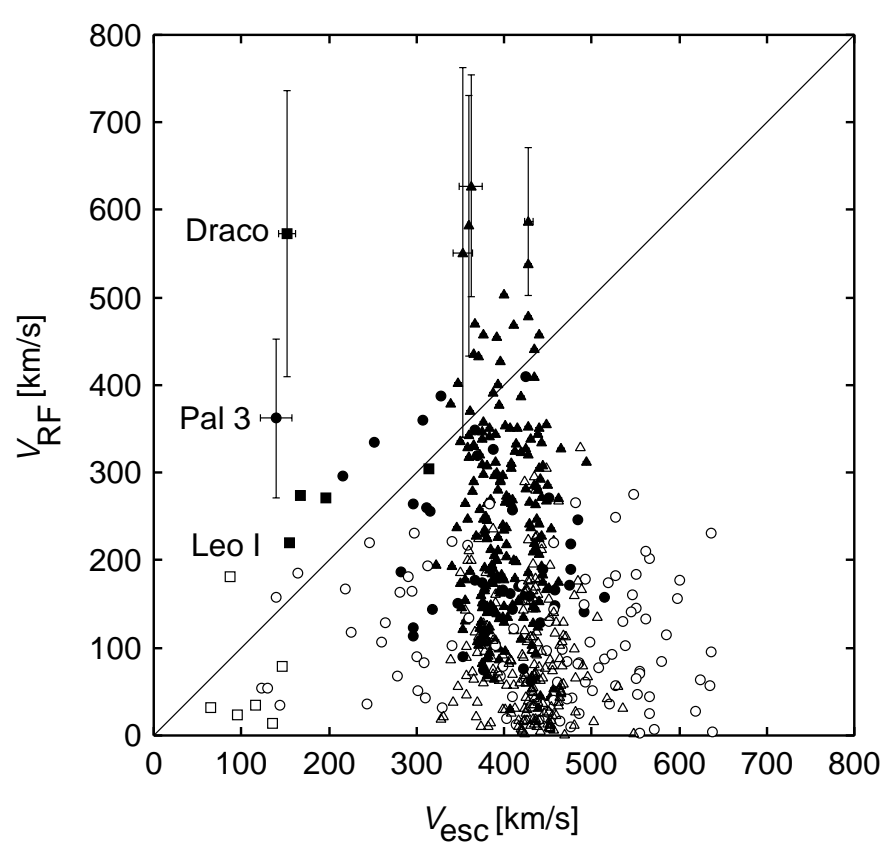

Fig. 4. The relation between escape velocities, $V_{\text {esc }}$, and space velocities, $V_{\mathrm{RF}}$, for Model A with $a=20 \mathrm{kpc}$ and $V_{\mathrm{LSR}}=211 \mathrm{~km} \mathrm{~s}^{-1}$. In this case, the rotation curve declines with increasing radii, as shown in Fig. 2 (dashed line). Note that, if this situation were to apply, many of the sample objects would be unbound to the Galaxy.

tracers alone, as in the previous section. In this approach, a phase-space distribution function of tracers, $F$, is prescribed for a specifically chosen $\psi$, and the model parameters included in $F$ and $\psi$ are derived so as to reproduce the presently observed positions and velocities of the tracers in the (statistically) most significant manner. The optimal deduced parameters relevant to $\psi$ then allow us to estimate the total mass of the Galaxy. This method was originally proposed by Little \& Tremaine (1987), and further developed by Kochanek (1996) and WE99.

Based on the results presented in the previous section, we take Model A with spherical symmetry as the mass distribution of the Galaxy, which is sufficient for the following analysis. For the sake of simplicity, and also for ease of comparison with the previous studies by Kochanek (1996) and WE99, the phasespace distribution function is taken to have the same anisotropic form as that adopted in these studies. That is, it depends on the binding energy per unit mass, $\varepsilon\left(\equiv \psi-v^{2} / 2\right)$, and the angular momentum per unit mass, $l$, in the following way:

$F(\varepsilon, l)=l^{-2 \beta} f(\varepsilon)$,

where

$$
\begin{aligned}
f(\varepsilon)= & \frac{2^{\beta-3 / 2}}{\pi^{3 / 2} \Gamma[m-1 / 2+\beta] \Gamma[1-\beta]} \frac{\mathrm{d}}{\mathrm{d} \varepsilon} \\
& \times \int_{0}^{\varepsilon} \mathrm{d} \psi \frac{\mathrm{d}^{m} r^{2 \beta} \rho_{\mathrm{s}}}{\mathrm{d} \psi^{m}}(\varepsilon-\psi)^{\beta-3 / 2+m},
\end{aligned}
$$

where $\rho_{\mathrm{s}}$ is the tracer density distribution, $\Gamma$ is the gamma function, and $m$ is an integer whose value is chosen such that the integral in Eq. (9) converges (e.g., Dejonghe 1986; Kochanek 1996). In the spherical model, this form of the distribution

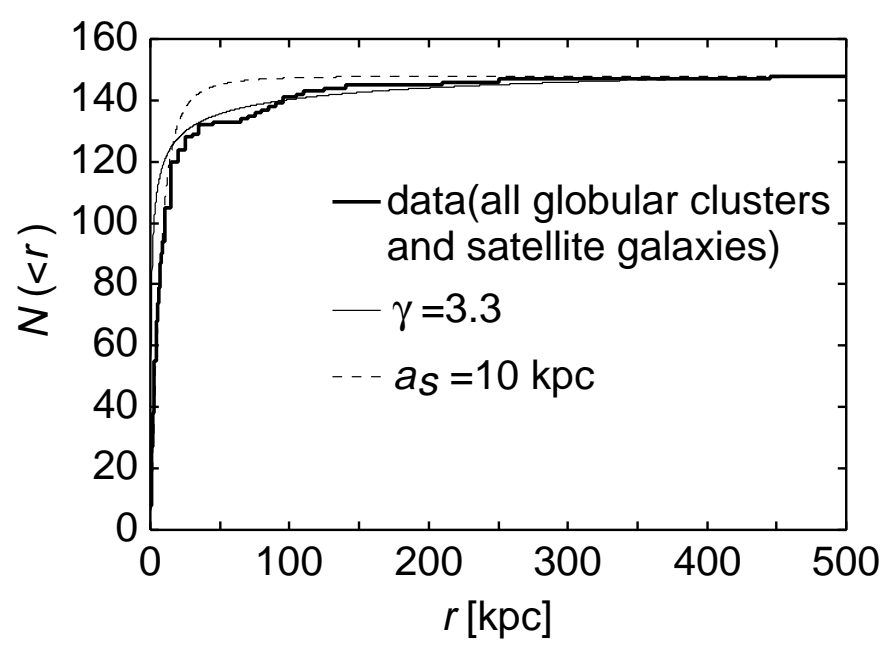

Fig. 5. Cumulative number distribution, $N(<r)$, of the distances of globular clusters and satellite galaxies (solid histogram) in comparison with model distributions (continuous dashed and solid lines). See the text for additional information.

function yields equal velocity dispersions in the orthogonal angular directions, $\left\langle v_{\theta}^{2}\right\rangle=\left\langle v_{\phi}^{2}\right\rangle$, and a constant anisotropy $\beta=1-<v_{\theta}^{2}>/<v_{\mathrm{r}}^{2}>$ everywhere in the Galaxy. Our choice of $m=2$ in Eq. (9) (to be in accord with the WE99 work) limits the allowed range for the velocity anisotropy to $-1.5 \leq \beta \leq 1$ when proper motion data are considered, while the use of radial velocities alone sets no limit for tangential anisotropy $[-\infty, 1]$.

For $\rho_{\mathrm{s}}$, we consider WE99's two models: (a) Shadow tracers following the mass density distribution obtained from Model A (Eq. (4)), and (b) a power-law distribution as a function of $r$. The shadow-tracer model is given as:

$\rho_{\mathrm{s}}(r) \propto \frac{a_{\mathrm{s}}^{2}}{r^{2}\left(r^{2}+a_{\mathrm{s}}^{2}\right)^{3 / 2}}$,

where $a_{\mathrm{s}}$ is the scale length. The power-law model with index $\gamma$ is given as:

$\rho_{\mathrm{s}}(r) \propto \frac{1}{r^{\gamma}}$

It should be noted that, since shadow tracers may be truncated at the distance below the scale length of the mass distribution, the scale length of the tracers, $a_{\mathrm{s}}$, is generally different from the scale length of the Galaxy's mass, $a$.

Using the 27 objects (satellite galaxies and globular clusters) at $r>20 \mathrm{kpc}$, WE99 derived $a_{\mathrm{s}}=100 \mathrm{kpc}$ and $\gamma=3.4$ as the best fitting parameters for their spatial distribution. We re-examine $a_{\mathrm{s}}$ and $\gamma$ using our sample of all satellite galaxies and globular clusters. Note that the FHB stars are excluded in this determination of $a_{\mathrm{s}}$ and $\gamma$, as they have not (yet) been completely surveyed over the Galactic volume. We obtain $a_{\mathrm{s}}=10 \mathrm{kpc}$ and $\gamma=3.3$ as the best-fit values, based on a simple $\mathrm{K}-\mathrm{S}$ test of the observed vs. predicted distribution functions (see Fig. 5). If we exclude the globular clusters at $r \leq 10 \mathrm{kpc}$, for which the spherical symmetry assumption may be questionable due to the presence of the disk globular clusters, we obtain $a_{\mathrm{s}}=50 \mathrm{kpc}$ and $\gamma=3.4$. Thus, $a_{\mathrm{s}}$ depends sensitively on the adopted range of radius (i.e., on the selection of the sample), 
whereas $\gamma$ basically remains unchanged. Therefore, we focus our attention on the results obtained from the power-law representation for the tracer population; the shadow-tracer population is also examined for the purpose of comparison with WE99. To see the dependence of the mass estimate on these parameters, we obtain estimates for two values of $\gamma(3.4$ and 4.0) and $a_{\mathrm{s}}(100 \mathrm{kpc}$ and the scale length of the mass distribution, a), respectively. We note that the FHB stars are also expected to follow a power-law form with $\gamma \simeq 3.4$, as inferred from other halo field stars (e.g., Preston et al. 1991; Chiba \& Beers 2001).

We calculate the likelihood of a particular set of model parameters (the scale length of the mass distribution, $a$, and the anisotropy parameter, $\beta$ ) given the positions, $r_{i}$, and radial velocities, $v_{r i}$, or space velocities, $v_{i}$, using Bayes' theorem. The probability that the model parameters take the values $a$ and $\beta$, given the data $\left(r_{i}, v_{(r)}\right)$ and prior information $I$, is:

$P\left(a, \beta \mid r_{i}, v_{(r) i}, I\right)=\frac{1}{N} P(a) P(\beta) \prod_{i=1}^{N} P\left(r_{i}, v_{(r)} \mid a, \beta\right)$,

where $N$ is the normalization factor (Kochanek 1996; WE99). The probabilities $P(a)$ and $P(\beta)$ denote the prior probability distributions in $a$ and $\beta$, respectively. Here, $P\left(r_{i}, v_{(r) i} \mid a, \beta\right)$ corresponds to the probability of finding an object at position $r_{i}$ moving with radial velocity $v_{(r) i}$, or space velocity $v_{i}$, for a particular set of model parameters $a$ and $\beta$. The complete expressions for $P\left(r_{i}, v_{(r) i} \mid a, \beta\right)$ are shown in Table 1 of WE99. To calculate this probability for the objects with full space velocities, we take into account their large errors relative to radial velocities alone (due to the observed proper-motion and assumed distance errors), by multiplying by an error convolution function of the form:

$$
P\left(r_{i}, v_{i} \mid a, \beta\right)=\int_{\times P\left(r_{i}, v_{i, \text { obs }}\left(v_{\alpha}, v_{\delta}\right) \mid a, \beta\right),} \mathrm{d} v_{\alpha} \mathrm{d} v_{\delta} E_{1}\left(v_{\alpha}\right) E_{1}\left(v_{\delta}\right)
$$

where $\left(v_{\alpha}, v_{\delta}\right)$ are the tangential velocities along the right ascension and declination coordinates, respectively, and $E_{1}$ is the Lorentzian error convolution function, defined as:

$E_{1}(v)=\frac{1}{\sqrt{2} \pi \sigma_{1}} \frac{2 \sigma_{1}^{2}}{2 \sigma_{1}^{2}+\left(v-v_{\mathrm{obs}}\right)^{2}}$,

where $\sigma_{1}$ is defined as $\sigma_{1}=0.477 \sigma$ for the calibrated error estimate $\sigma$ (see WE99).

The prior probability in the velocity anisotropy, $\beta$, is taken to be of the form $P(\beta) \propto 1 /(3-2 \beta)^{n}$, where $n=0$ and 2 correspond to a uniform prior and a uniform energy prior, respectively (Kochanek 1996; WE99). Larger values of $n$ yield a larger weight towards radial anisotropy. For the prior probability in $a, P(a)$, we adopt $1 / a$ and $1 / a^{2}$ (WE99).

Using the routine AMOEBA in Numerical Recipes (Press et al. 1992), we search for a set of model parameters, $a$ and $\beta$, that maximize the probability $P\left(a, \beta \mid r_{i}, v_{(r)}, I\right)$. The total mass of the Galaxy, $M$, is then derived from the parameter $a$.

\subsection{Results}

Initially, we apply the Bayesian likelihood method, making use of only the radial velocities of the objects, setting aside for the moment the available proper-motion information. Specifically, we focus on the difference in the mass estimate arising from the presence or absence of Leo I. Figure 6 shows the likelihood contours in the mass-anisotropy $(M-\beta)$ plane for the case of a power-law tracer population with $\gamma=3.4$, where $\beta$ is limited to the range of $-1.5 \leq \beta \leq 1$. The solid and dashed lines denote the presence and absence of Leo I, respectively. As is evident, the mass estimate sensitively depends on whether or not Leo I is bound to the Galaxy, as has been noted in previous studies. Inclusion of Leo I yields a likely total mass that is an order of magnitude greater than the case without Leo I. Over the range of $\beta$ we consider, the most likely value of $M$, with Leo I included, is $21.0 \times 10^{11} M_{\odot}$, corresponding to a scale length $a=185 \mathrm{kpc}$, whereas excluding Leo I yields $M=9.6 \times 10^{11} M_{\odot}$, and $a=85 \mathrm{kpc}$. We note that the role of Leo I in the Galaxy's mass estimate is also understandable from the escape-velocity argument; if only the sample radial velocities are taken into account, Leo I alone determines the best-fit boundary line $V_{\mathrm{RF}}=V_{\mathrm{esc}}$ in the $V_{\mathrm{RF}}$ vs. $V_{\mathrm{esc}}$ diagram (Fig. 3).

As is seen in Fig. 6, the high-probability region is biased toward the line $\beta=-1.5$. This bias arises from the specific form of the phase-space distribution function, $F(\varepsilon, l)$, given in Eq. (8), where the probability $P\left(a, \beta \mid r_{i}, v_{(r) i}, I\right)$ is high at large $F$. We plot $F$ in Fig. 7 for a set of $r$ and $\beta$ (solid and dotted lines for $\beta=-1$ and 1 , respectively). It follows that $F$ at high $\varepsilon$ is larger for smaller $\beta$, whereas $F$ at low $\varepsilon$ is larger for larger $\beta$. The range of $\varepsilon$ corresponding to these two different cases depends on $r$, as can be deduced from the comparison between panels a and $b$ in Fig. 7. Since our sample objects are mainly distributed in the region of higher $\varepsilon$ (solid histograms for the sample with radial velocities), the probability is highest at smallest $\beta$.

Following the above experiments, we drop the lower bound of -1.5 for $\beta$, and search for the maximum probability at smaller $\beta$. No maximum is found up to $\beta=-20$, although the large discrepancy in $M$ between the cases with and without Leo I remains. When we confine ourselves to the sample at $r>10 \mathrm{kpc}$, there exists a maximum probability at $\beta=-2.75$ (with Leo I), with a corresponding mass $32.0 \times 10^{11} M_{\odot}$. For the sample at $r>20 \mathrm{kpc}$, we obtain $11.4 \times 10^{11} M_{\odot}$ at $\beta=0.8$. This clearly suggests that the best-fitting $\beta$, obtained from the analysis when only radial velocities are considered, is rather sensitive to the range of $r$ employed in the sample selection. This in turn affects the number distribution, $N(\varepsilon)$, which is relevant to the likely range of $F$ (Fig. 7).

With these unavoidable limitations of the present sample in mind, Table 2 summarizes the likelihood results for the limited range of $-1.5 \leq \beta \leq 1$, obtained for power-law and shadow tracers, using a variety of different priors on $a$ and $\beta$. The most likely value of $\beta$ is -1.5 for all cases, for the reasons described above. We note that the current mass estimate is rather insensitive to the $\beta$ prior. As the $\beta$ prior decreases, the estimated mass generally increases, and the best-fitting $\beta$ decreases, because the small $\beta$ prior is biased toward more tangentially anisotropic velocity distributions than is the large $\beta$ prior. However, since most of our sample have high $\varepsilon$, the best-fitting $\beta$ remains -1.5 regardless of whether we adopt the uniform prior or 
Table 2. Likelihood results for only the radial velocities.

\begin{tabular}{|c|c|c|c|c|c|c|c|c|}
\hline$\gamma$ or $a_{s}$ & $a$ prior & $\beta$ prior & Leo I & best $\beta$ & best $a(\mathrm{kpc})$ & best $M^{\mathrm{a}}$ & $M(<50 \mathrm{kpc})^{\mathrm{a}}$ & $M(<100 \mathrm{kpc})^{\mathrm{a}}$ \\
\hline \multicolumn{9}{|c|}{ Power-law Tracers } \\
\hline \multirow[t]{2}{*}{$\gamma=3.4$} & $1 / a^{2}$ & Energy & Yes & -1.5 & 185 & 21.0 & 5.4 & 9.9 \\
\hline & & & No & -1.5 & 85 & 9.6 & 4.9 & 7.3 \\
\hline \multirow[t]{2}{*}{$\gamma=3.4$} & $1 / a$ & Energy & Yes & -1.5 & 210 & 24.0 & 5.5 & 10.0 \\
\hline & & & No & -1.5 & 95 & 11.0 & 5.0 & 7.8 \\
\hline \multirow[t]{2}{*}{$\gamma=3.4$} & $1 / a^{2}$ & Uniform & Yes & -1.5 & 185 & 21.0 & 5.4 & 9.9 \\
\hline & & & No & -1.5 & 85 & 9.6 & 4.9 & 7.3 \\
\hline \multirow[t]{2}{*}{$\gamma=4.0$} & $1 / a^{2}$ & Energy & Yes & -1.5 & 195 & 22.0 & 5.5 & 10.0 \\
\hline & & & No & -1.5 & 90 & 10.0 & 4.9 & 7.6 \\
\hline \multicolumn{9}{|c|}{ Shadow Tracers } \\
\hline \multirow[t]{2}{*}{$a_{s}=100$} & $1 / a^{2}$ & Energy & Yes & -1.5 & 210 & 24.0 & 5.5 & 10.0 \\
\hline & & & No & -1.5 & 85 & 9.6 & 4.9 & 7.3 \\
\hline \multirow[t]{2}{*}{$a_{s}=100$} & $1 / a$ & Energy & Yes & -1.5 & 240 & 27.0 & 5.5 & 10.0 \\
\hline & & & No & -1.5 & 95 & 11.0 & 5.0 & 7.8 \\
\hline \multirow[t]{2}{*}{$a_{s}=100$} & $1 / a^{2}$ & Uniform & Yes & -1.5 & 210 & 24.0 & 5.5 & 10.0 \\
\hline & & & No & -1.5 & 85 & 9.6 & 4.9 & 7.3 \\
\hline \multirow[t]{2}{*}{$a_{s}=a$} & $1 / a^{2}$ & Energy & Yes & -1.5 & 205 & 23.0 & 5.5 & 10.0 \\
\hline & & & No & -1.5 & 90 & 10.0 & 4.9 & 7.6 \\
\hline
\end{tabular}

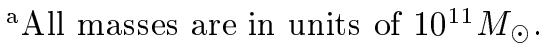

the uniform-energy prior for $\beta$. This property makes the mass estimate insensitive to the $\beta$ prior.

Now we apply the Bayesian likelihood method to the subsample of objects with both radial velocities and proper motions available, and consider the derived space motions. In contrast to the above case, where we used radial velocities alone, we find that the maximum probability within the range of $\beta$ we consider is now bounded (Fig. 8a). This may be caused by the characteristic distribution of $\varepsilon$ for the sample with full space motions, as shown in Fig. 7 (dotted histogram). This figure shows that there exists a larger fraction of low- $-\varepsilon$ stars than are found in the sample with radial velocities alone (solid histograms), so a larger $\beta$ is preferred to achieve a larger $F$. The mass estimate in this case is quite insensitive to the presence or absence of Leo I. Figure 8b shows the probabilities, as a function of $M$, with a fixed value of $\beta=-1.25$, for the case of a power-law tracer population with $\gamma=3.4$. Solid and dashed lines denote the probabilities with and without Leo I, respectively. As is evident, the agreement between both probabilities is significantly improved compared to the case when the radial velocities are considered alone (Fig. 6b). When Leo I is included, the most likely value of the total mass, $M$, and the scale length, $a$, are $25.0 \times 10^{11} M_{\odot}$ and $225 \mathrm{kpc}$, respectively. Excluding Leo I yields $M=18.0 \times 10^{11} M_{\odot}$ and $a=160 \mathrm{kpc}$. Table 3 summarizes the various results obtained when the proper motions of the objects are considered. This table illustrates that, for all cases, the mass of the Galaxy obtained when including Leo I is in good agreement with that obtained without Leo I. Also, the mass estimate depends only weakly on the index $\gamma$, unknown prior probabilities for $a$ and $\beta$, as well as on the range of $r$ used in the sample selection, resulting in small changes in the mass estimates over a range of only a few times $10^{11} M_{\odot}$.

To estimate the typical errors in this mass determination that are associated with the measurement errors of the 561 tracers we have analyzed, we have conducted Monte Carlo simulations, adopting the assumptions that typical errors in the distances and radial velocities are $10 \%$, and $10 \mathrm{~km} \mathrm{~s}^{-1}$, re-

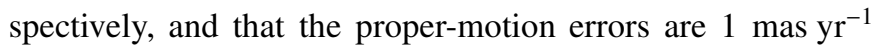

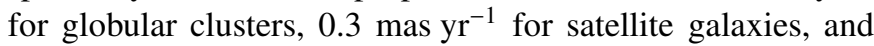

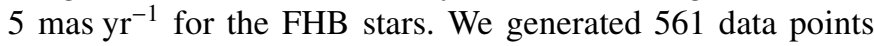
(including Leo I) drawn from Gaussian distribution functions centered on the observational data, and with dispersions set to the above typical errors. Given a true mass $M$, or scale length $a$ (where we use $M=2.3 \times 10^{12} M_{\odot}$ with $a=200 \mathrm{kpc}$ ), and prior probabilities for $a$ and $\beta\left(1 / a^{2}\right.$ and the uniform-energy prior, respectively), we calculate the most likely mass, $M^{\prime}$, and compare it with an input true mass. Figure 9 shows the distribution of the discrepancy between $M^{\prime}$ and $M, 100 \times\left(M^{\prime}-M\right) / M$, obtained from 1000 realizations. The error distribution in the current mass estimate has a mean value shifted downward by $20 \%$, and a dispersion of half-width $20 \%$. These values suggest that one might adopt an estimate of the systematic error on the order of $20 \%$, and a random error of $\pm 20 \%$. Exclusion of Leo I does not influence the magnitude of these errors. It is worth noting that WE99 obtained roughly $\sim 100 \%$ systematic errors, and $\sim 90 \%$ random errors in their mass estimate, which was based on about 30 data points. The significant 
Table 3. Likelihood results for the full space velocities.

\begin{tabular}{|c|c|c|c|c|c|c|c|c|}
\hline$\gamma$ or $a_{s}$ & $a$ prior & $\beta$ prior & Leo I & best $\beta$ & best $a(\mathrm{kpc})$ & best $M^{\mathrm{a}}$ & $M(<50 \mathrm{kpc})^{\mathrm{a}}$ & $M(<100 \mathrm{kpc})^{\mathrm{a}}$ \\
\hline \multicolumn{9}{|c|}{ Power-law Tracers } \\
\hline \multirow[t]{2}{*}{$\gamma=3.4$} & $1 / a^{2}$ & Energy & Yes & -1.25 & 225 & 25.0 & 5.5 & 10.0 \\
\hline & & & No & -1.25 & 160 & 18.0 & 5.4 & 9.6 \\
\hline \multirow[t]{2}{*}{$\gamma=3.4$} & $1 / a$ & Energy & Yes & -1.25 & 255 & 29.0 & 5.5 & 10.0 \\
\hline & & & No & -1.25 & 175 & 20.0 & 5.4 & 9.8 \\
\hline \multirow[t]{2}{*}{$\gamma=3.4$} & $1 / a^{2}$ & Uniform & Yes & -1.25 & 225 & 25.0 & 5.5 & 10.0 \\
\hline & & & No & -1.25 & 160 & 18.0 & 5.4 & 9.6 \\
\hline \multirow[t]{2}{*}{$\gamma=4.0$} & $1 / a^{2}$ & Uniform & Yes & -1.35 & 245 & 28.0 & 5.5 & 10.0 \\
\hline & & & No & -1.35 & 175 & 20.0 & 5.4 & 9.8 \\
\hline \multicolumn{9}{|c|}{ Shadow Tracers } \\
\hline \multirow[t]{2}{*}{$a_{s}=100$} & $1 / a^{2}$ & Energy & Yes & -1.25 & 290 & 33.0 & 5.5 & 11.0 \\
\hline & & & No & -1.25 & 200 & 21.0 & 5.5 & 10.0 \\
\hline \multirow[t]{2}{*}{$a_{s}=100$} & $1 / a$ & Energy & Yes & -1.25 & 350 & 37.0 & 5.6 & 11.0 \\
\hline & & & No & -1.25 & 240 & 25.0 & 5.5 & 10.0 \\
\hline \multirow[t]{2}{*}{$a_{s}=100$} & $1 / a^{2}$ & Uniform & Yes & -1.25 & 290 & 33.0 & 5.5 & 11.0 \\
\hline & & & No & -1.25 & 200 & 21.0 & 5.5 & 10.0 \\
\hline \multirow[t]{2}{*}{$a_{s}=a$} & $1 / a^{2}$ & Energy & Yes & -1.3 & 315 & 35.0 & 5.6 & 11.0 \\
\hline & & & No & -1.3 & 220 & 25.0 & 5.5 & 10.0 \\
\hline
\end{tabular}

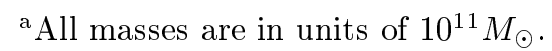

improvement of our mass estimate is mainly due to our consideration of a much larger data set that includes several hundred FHB stars.

As shown in Table 3, the most likely estimated total mass depends on model assumptions at a level of a few times $10^{11} M_{\odot}$. When the model is fixed, the current large data set allows us to limit both systematic and random errors to a level of about 20\%. If we follow WE99's procedure for the adoption of the most likely total mass, i.e., if we adopt the mass estimate that provides the smallest difference between the masses obtained with Leo I and without Leo I, we obtain $2.5_{-1.0}^{+0.5} \times 10^{12} M_{\odot}$ (Leo I included) and $1.8_{-0.7}^{+0.4} \times 10^{12} M_{\odot}$ (Leo I excluded). On the other hand, the mass estimate within the distance of the LMC $(50 \mathrm{kpc})$ is quite robust, covering the narrow range 5.4 to $5.5 \times 10^{11} M_{\odot}$.

\section{Discussion and concluding remarks}

We have placed new limits on the mass of the Galaxy, based on a newly assembled set of halo objects with the latest available proper-motion data. First, the comparison of their space velocities with the escape velocities at their estimated distances allowed us to show that the mass limits we obtained depend on neither the presence or absence of Leo I, nor on the large velocity errors for Draco and Pal 3; a possible a lower limit on the total mass of the Galaxy is about $\sim 2.2 \times 10^{12} M_{\odot}$. Secondly, a Bayesian likelihood approach has been used to derive a total mass estimate for the Galaxy that is insensitive to the presence or absence of Leo I, at least when proper motions are taken into account. Although the best mass estimate obtained from this approach depends somewhat on model assumptions (prior probabilities for $a$ and $\beta$ and possibly the shape of $F$, see below), the resultant systematic change of the total mass is confined to within a few times $10^{11} M_{\odot}$. The most likely total mass of the Galaxy we derive is $2.5_{-1.0}^{+0.5} \times 10^{12} M_{\odot}$. This is in good agreement with the total mass obtained by WE99 $\left(1.9_{-1.7}^{+3.6} \times 10^{12} M_{\odot}\right)$ and that obtained from other methods (e.g., Peebles $\left.1995,2 \times 10^{12} M_{\odot}\right)$. Since the size of our tracer sample is significantly larger than used in previous studies, both systematic and random errors are reduced to a great extent. We note that consideration of the numerous FHB stars plays a vital role in this mass estimate, as demonstrated in Sect. 3.

It is also worth noting that, if we fix the mass of the Galaxy equal to our most likely mass estimate, there is insufficient matter present to gravitationally bind the LMC, if we adopt the recent proper-motion measurement by Anguita et al. (2000). These authors reported rather high proper motions, $\left(\mu_{\alpha} \cos \delta, \mu_{\delta}\right)=(+1.7 \pm 0.2,+2.9 \pm 0.2)$, compared to previous measurements, $\left(\mu_{\alpha} \cos \delta, \mu_{\delta}\right)=(+1.94 \pm 0.29,-0.14 \pm 0.36)$ (Kroupa \& Bastian 1997). Thus their results need confirmation from other studies ${ }^{2}$.

The current work also implies that the Galactic rotation curve at outer radii, $R>R_{\odot}$, does not decline out to at least $R \sim 20 \mathrm{kpc}$ (as long as local disturbances to circular motions, such as warping motions and/or non-axisymmetric motions, are ignored). As illustrated in Fig. 2, a declining rotation curve

\footnotetext{
2 Since we finished our analysis here, a new study by Pedreros et al. (2002) has been published (using the same method as Anguita el al. 2000), that reaches the same conclusion as Kroupa \& Bastian (1997).
} 
(a)

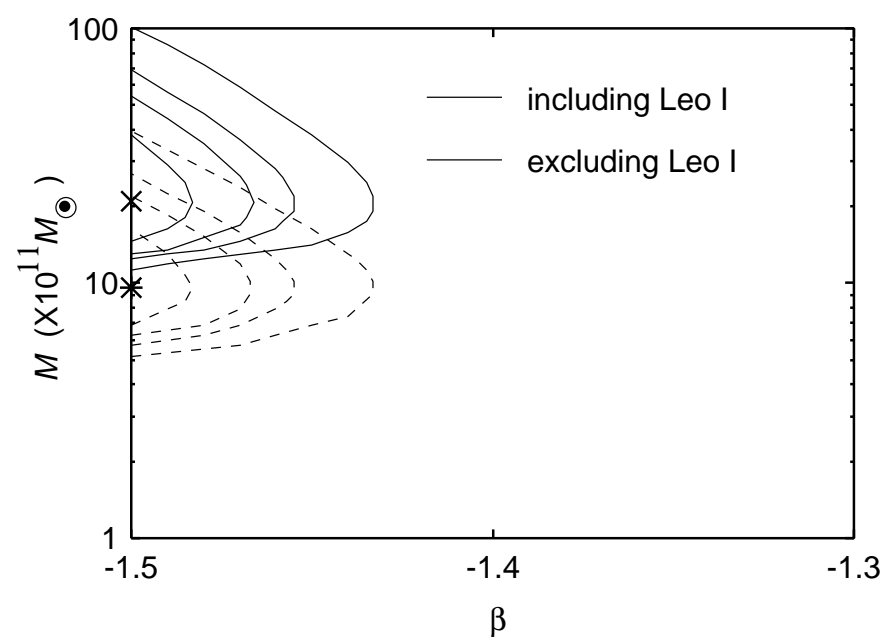

(b)

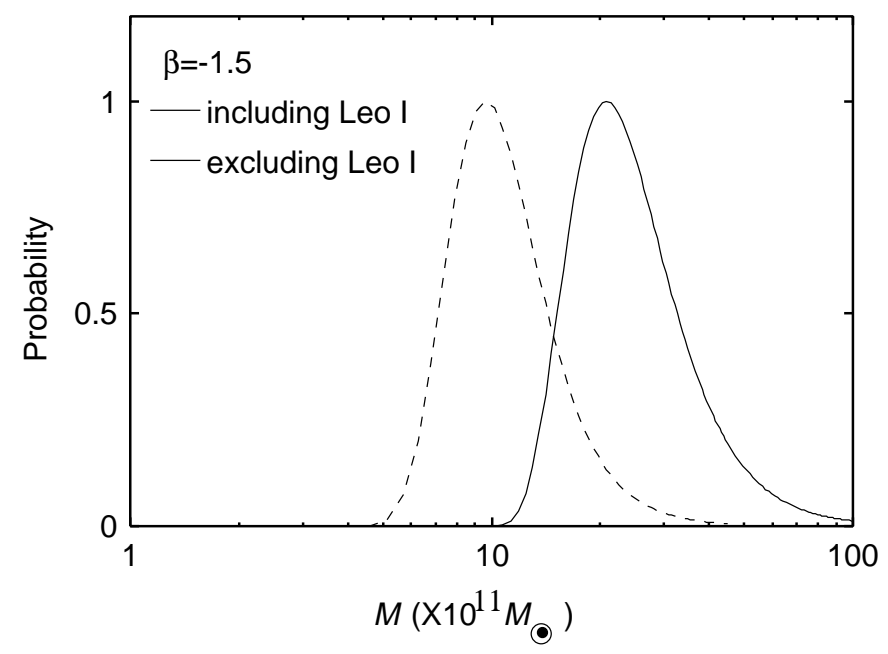

Fig. 6. a) Likelihood contours in the plane of the mass, $M$, and velocity anisotropy, $\beta$, obtained from an analysis using only radial velocities. The solid and dashed curves show the results including Leo I and excluding Leo I, respectively; the cross and the asterisk show the maxima of the probabilities for each case. Contours are plotted at heights of $0.32,0.1,0.045$, and 0.01 of the peak height. The spatial distribution of a tracer population is assumed to follow a power-law form with $\gamma=3.4$. b) Probabilities of the mass $M$ at $\beta=-1.5$, including Leo I (solid line) and excluding Leo I (dashed line).

corresponding to $a=20 \mathrm{kpc}$ and $V_{\mathrm{LSR}}=211 \mathrm{~km} \mathrm{~s}^{-1}$ fails to bind many sample objects to the Galaxy. The smallest possible value for $a$ to bind all objects in the isothermal-like density distribution (Eq. (4)) is $a=195 \mathrm{kpc}$, yielding $V_{\mathrm{LSR}} \simeq 220 \mathrm{~km} \mathrm{~s}^{-1}$.

In a more general context, the detailed shape of the rotation curve at and beyond $R=R_{\odot}$ reflects the interplay between the disk and halo mass distributions, as this region is located near the boundary of both components. Thus, determining the rotation curve at $R_{\odot} \lesssim R \lesssim 15 \mathrm{kpc}$ will set useful limits on the mass distribution in the inner parts of the Galaxy. Indeed, the Japanese project VLBI Exploration of Radio Astrometry (VERA) will be able to determine both inner and outer rotation curves from the measurement of trigonometric parallaxes and (a)

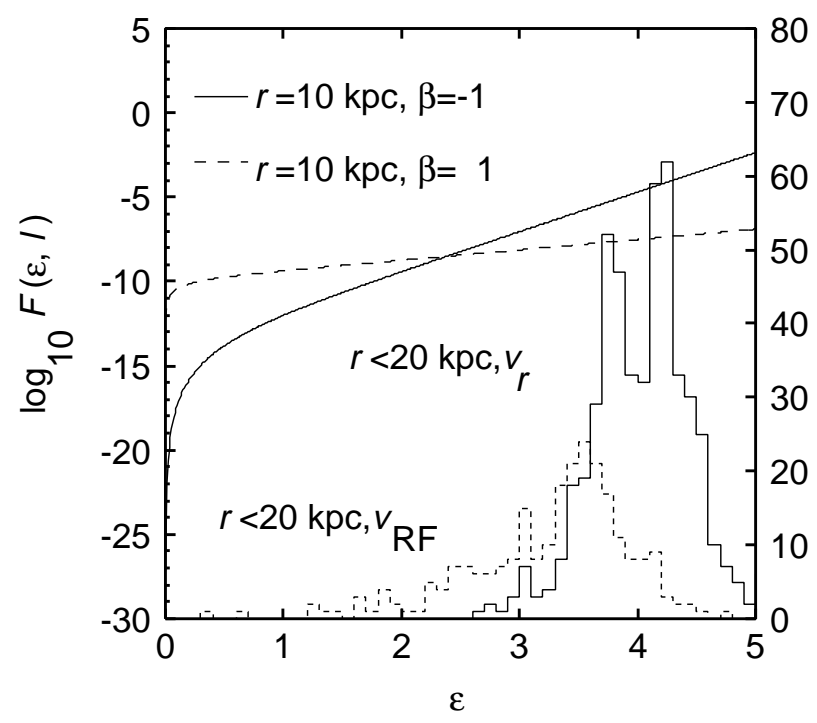

(b)

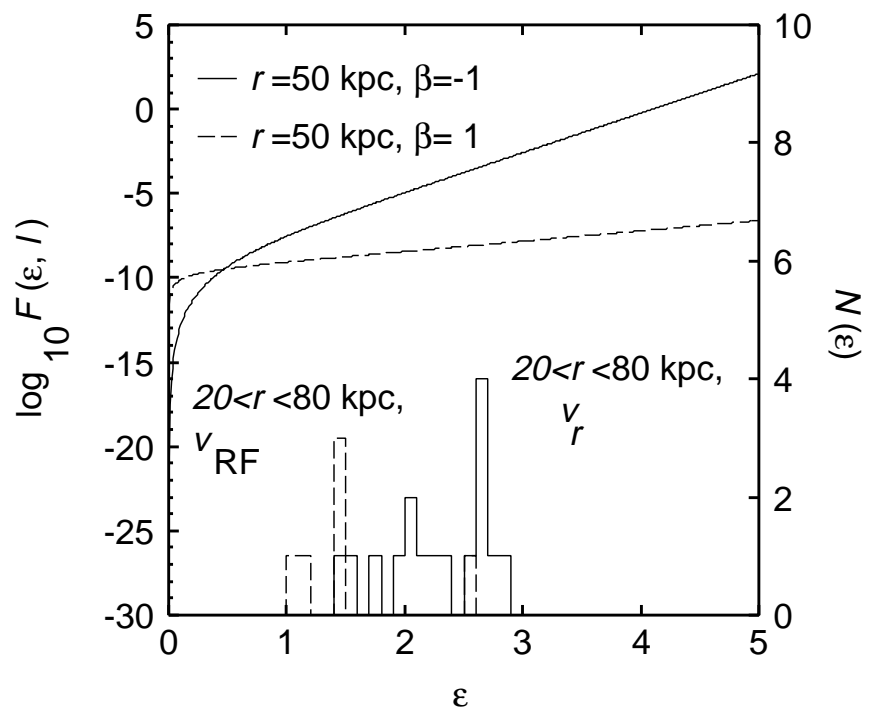

Fig. 7. The distribution function, $F$, for $\beta=-1$ (solid lines) and $\beta=1$ (dotted lines), at $r=10 \mathrm{kpc}($ panel a)) and $r=50 \mathrm{kpc}$ (panel b)). Also plotted are the number distributions $N(\varepsilon)$ of the stars when $a=$ $200 \mathrm{kpc}$, where dotted and solid histograms denote the sample with and without available proper motions, respectively. The range of $r$ for plotting $N(\varepsilon)(r<20 \mathrm{kpc}$ for panel a) and $20<r<80 \mathrm{kpc}$ for panel b)) is chosen to approximately match that for $F$.

proper motions of astronomical maser sources that are widely distributed in the Galactic disk (Sasao 1996; Honma et al. 2000). VERA will reach unprecedented astrometric precision, $\sim 10 \mu$ as, and will yield precise determinations of the Galactic constants $R_{\odot}$ and $V_{\mathrm{LSR}}$. We note that whatever results are derived for the rotation curve, the total mass of the Galaxy ought to be larger than $10^{12} M_{\odot}$, in order to bind the more distant stellar objects.

Our estimate for the mass of the Galaxy inside $50 \mathrm{kpc}$, i.e., within the distance of the $\mathrm{LMC}$, is $5.5_{-0.3}^{+0.0} \times 10^{11} M_{\odot}$ (Leo I included) and $5.3_{-0.4}^{+0.1} \times 10^{11} M_{\odot}$ (Leo I excluded). The error estimates are calculated from the maximum and minimum 
(a)

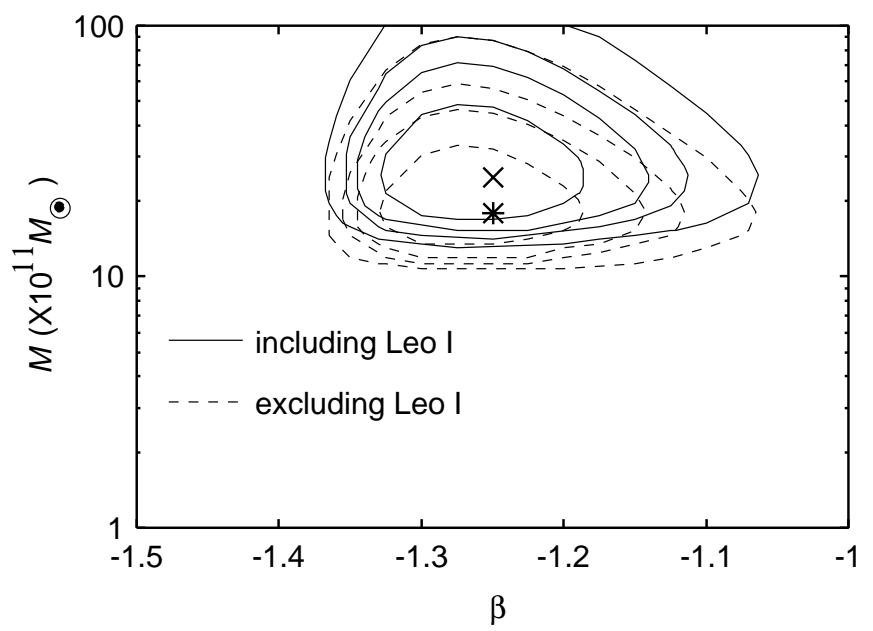

(b)

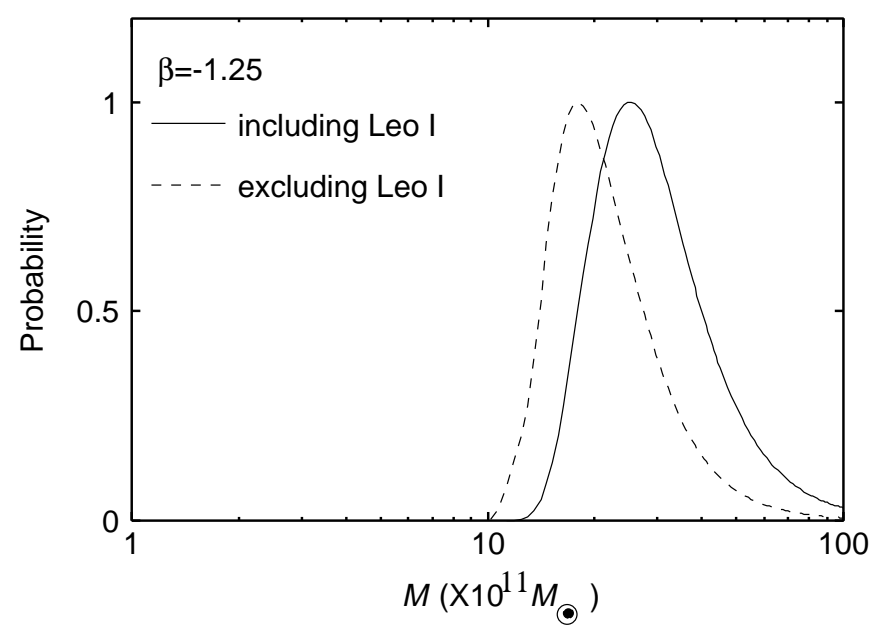

Fig. 8. a) Likelihood contours in the plane of the mass, $M$, and velocity anisotropy, $\beta$, obtained from an analysis that uses both radial velocities and proper motions. Solid and dashed curves show the results including Leo I and excluding Leo I, respectively; the cross and the asterisk show the maxima of the probabilities for each case. Contours are plotted at heights of $0.32,0.1,0.045$, and 0.01 of the peak height. The spatial distribution of a tracer population is assumed to follow a power-law form with $\gamma=3.4$. b) Probabilities of the mass $M$ at the best-fitting $\beta$ of -1.25 , including Leo I (solid line) and excluding Leo I (dashed line).

values of the total mass. Thus, about $24 \%$ of the total mass of the Galaxy resides within $r \leq 50 \mathrm{kpc}$. This implies that the possibility of brown dwarfs as the origin of the microlensing events toward the LMC may be excluded, because it requires a much smaller mass inside $50 \mathrm{kpc}, \sim 1.3 \times 10^{11} M_{\odot}$ (Honma \& Kan-ya 1998). Our result is also in good agreement with the recent statistics of the microlensing events obtained from analysis of the 5.7-year baseline of photometry for 11.9 million stars in the LMC (Alcock et al. 2000), showing the absence of shortduration lensing events by brown dwarfs. However, the most recent work has suggested that perhaps one of the microlensing events is actually caused by a nearby low-mass star in the

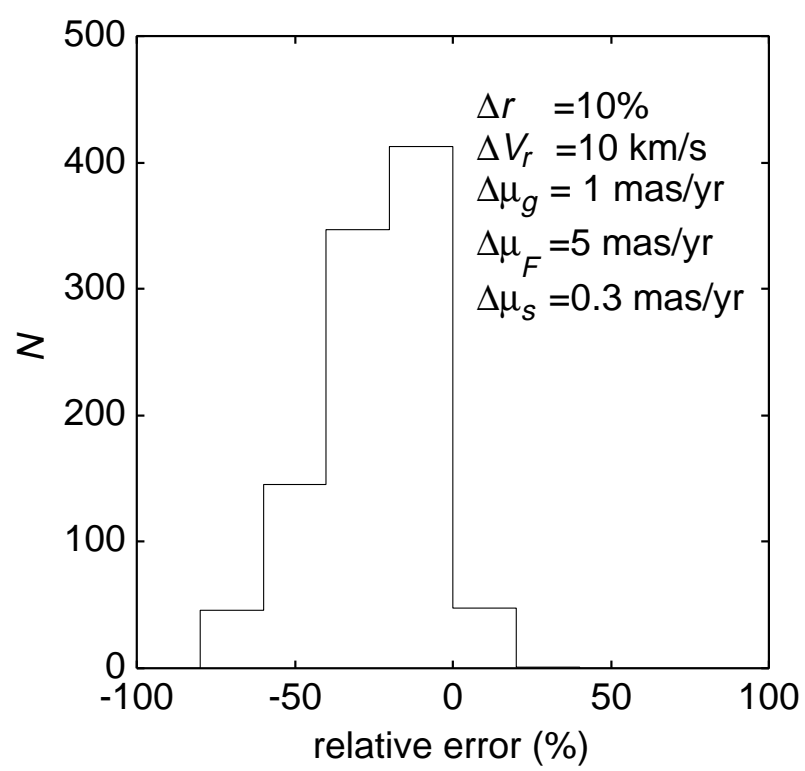

Fig. 9. An approximate error distribution of the mass estimate caused by the typical measurement errors of the data. The abscissa denotes the relative error in mass, $100 \times\left(M^{\prime}-M\right) / M$, where $M^{\prime}$ is the mass calculated by a Monte Carlo method and $M$ is the input true value. See text for more details.

Galactic disk (Alcock et al. 2001). More direct observations for identifying lensing objects are required to settle this issue.

Once the total mass of the Galaxy is fixed, it is possible to place a useful constraint on the mass of the Local Group. Most of the mass in the Local Group is concentrated in M 31 and the Galaxy. The total mass of M 31 can be estimated from the positions and radial velocities of its satellite galaxies, globular clusters, and planetary nebulae (Evans \& Wilkinson 2000; Côté et al. 2000; Evans et al. 2000). If we take it to be $1.2_{-0.6}^{+1.8} \times 10^{12} M_{\odot}$ (Evans \& Wilkinson 2000), the mass of the Local Group is $\sim 3.7 \times 10^{12} M_{\odot}$. This is in good agreement with the estimate by Schmoldt \& Saha (1998), (4-8) $\times 10^{12} M_{\odot}$, based on modified variational principles.

To set tighter limits on the total mass of the Galaxy we require more accurate proper-motion measurements for a greater number of objects at large Galactocentric distances. The highvelocity FHB stars in our sample (with apparent magnitudes $V<16$ ) that are responsible for setting the minimum mass of

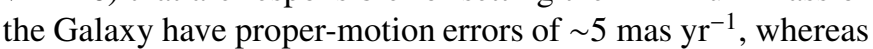
Draco and Pal 3 have much larger relative errors, comparable to their proper motions themselves (see Table 2). Indeed, both the Space Interferometry Mission (SIM: Unwin et al. 1997) and the Global Astrometry Interferometer for Astrophysics (GAIA: Lindegren \& Perryman 1996) will be able to provide more accurate proper motions for such high-velocity objects, as well as for numerous other distant tracers of the Galaxy's mass, up to a precision of a few $\mu$ as for targets with $V \leq 15$. This corresponds to an error of $\$ 10 \mathrm{~km} \mathrm{~s}^{-1}$ in the tangential velocity components for many distant objects, i.e., comparable to the error of their (presently determined) radial velocities. Furthermore, roughly half of our sample objects lack propermotion measurements altogether. To a great extent, the lack of proper-motion measurements (at least for southern sources) 
will be removed with the completion of the recently re-started Southern Proper Motion survey of van Altena and colleagues, as well as other efforts to substantially increase the numbers of stars with reasonably well-measured proper motions (e.g., UCAC1: Zacharias et al. 2000; UCAC2: Zacharias et al. 2001).

Further assembly of radial velocities for FHB stars, especially those at large $r$ (beyond distances where accurate groundbased proper motions can be obtained), is also of great importance for a number of reasons. First, as Fig. 3 demonstrates, large Galactocentric regions are characterized by small escape velocities. The current sample of FHB stars (because of their locations near the Sun) explore distances where the corresponding escape velocities are in the range of $500 \lesssim V_{\text {esc }} \lesssim$ $600 \mathrm{~km} \mathrm{~s}^{-1}$. More remote FHB stars, with distances in the range $10 \lesssim r \lesssim 50 \mathrm{kpc}$, will offer a further constraint on the total mass of the Galaxy by covering the range $400 \lesssim V_{\text {esc }} \lesssim 500 \mathrm{~km} \mathrm{~s}^{-1}$. Secondly, the assembly of samples of more distant FHB stars will enable exploration of the suggested change in velocity anisotropy from the inner to the outer halo (e.g., SommerLarsen et al. 1997), and better constrain its dependence on Galactocentric distance.

In exploring the Bayesian approach for mass estimates of the Galaxy, we have adopted a specific form of the phase-space distribution function $F$ (Eq. (8)) to facilitate comparison with previous studies. This procedure implicitly assumes that the velocity-anisotropy parameter, $\beta$, is constant everywhere in the Galactic volume. However, as noted by Sommer-Larsen et al. (1997), there is an indication that the velocity anisotropy of the halo may be mostly radial at $R \lesssim 20 \mathrm{kpc}$ and tangential at $R \gtrsim 20 \mathrm{kpc}$. If so, many of distant FHB stars, especially those at $R>20 \mathrm{kpc}$, play a crucial role in the determination of the global distribution of velocity anisotropy. Searches for a more realistic form of the phase-space distribution function, combined with a more elaborate likelihood method, are both worthy pursuits. Also, instead of exploring such a specific but realistic form of distribution function, a non-parametric method as proposed by Merritt \& Tremblay (1993) will be more useful if a much larger data set is available.

Moreover, the implicit assumption behind the current method, that the sample stars have random distributions in location and in their space motions, may not be well satisfied if the halo is largely dominated by coherent structures such as tidal streams (e.g., Ibata et al. 2001). Alternative approaches to obtaining mass limits using tidal streams (Johnston et al. 1999) are worth considering in such a case.

Fortunately, prospects are excellent for obtaining a rapid increase in the observational database of FHB stars with the required data. There already exists a substantial body of additional spectroscopy for FHB/A stars observed during the course of the HK survey of Beers and colleagues and the Hamburg/ESO Stellar survey (Christlieb et al. 2001), many of which also have available proper motions, or will soon, from completion of the SPM survey and/or other ground-based efforts. However, as was noted by Wilhelm et al. (1999a) (foreshadowed by Norris \& Hawkins 1991; Rodgers \& Roberts 1993, and references therein; Kinman et al. 1994; Preston et al. 1994), a substantial fraction (perhaps as high as 50\%) of high-latitude A-type stars are not FHB, but rather some (as yet undetermined) mixture of binaries and high-gravity stars (see Preston \& Sneden 2000). For some applications, such as estimates of the mass of the Galaxy that rely on space motions of tracers (and in turn on reasonably precise distance estimates of individual objects), confident separation of bonafide members of the FHB population from possible "contaminants" is crucial ${ }^{3}$. In the past, this has required that one obtain either Strömgren photometry and/or spectrophotometry (e.g., Kinman et al. 1994), broad-band $U B V$ photometry in combination with medium-resolution spectroscopy (e.g., Wilhelm et al. 1999 a), or reasonably high $S / N$, high-resolution spectroscopy (e.g., Preston \& Sneden 2000). All such endeavors are rather time intensive. However, Christlieb et al. (2002, priv. comm.) have been exploring means by which adequate separation of FHB stars from higher-gravity A-type stars might be accomplished directly from objective-prism spectra, such as those in the Hamburg/ESO stellar survey. Such methods, which look promising, would be most helpful in future investigations of this sort. Wide-field stellar surveys, such as those presently being carried out with the $6 \mathrm{dF}$ facility at the UK Schmidt Telescope, are capable of providing large numbers of radial velocities for FHB/A candidates, and are expected to contribute 5000-10000 suitable data over the course of the next few years.

Acknowledgements. We are grateful to B. Fuchs, R. B. Hanson, and I. Platais for assistance with the comparison of the Wilhelm et al. sample with the catalogs of STARNET, NPM1, and SPM 2.0, respectively. We also thank the members of the VERA team for several useful comments on this work. T.C.B. acknowledges partial support from grants AST-00 98508 and AST-00 98549 awarded by the U.S. National Science Foundation. T.C.B also would like to acknowledge the support and hospitality shown him during a sabbatical visit to the National Astronomical Observatory of Japan, funded in part by an international scholar award from the Japanese Ministry of Education, Culture, Sports, Science, and Technology, during which initial discussions of this work took place.

\section{References}

Alcock, C., Allsman, R. A., Alves, D. R., et al. 2000, ApJ, 542, 281

Alcock, C., Allsman, R. A., Alves, D. R., et al. 2001, Nature, 414, 617

Anguita, C., Loyola, P., \& Pedreros, M. H. 2000, AJ, 120, 845

Beers, T. C., Chiba, M., Yoshii, Y., et al. 2000, AJ, 119, 2866

Binney, J. J., \& Tremaine, S. 1987, Galactic Dynamics (Princeton Univ. Press, Princeton)

Carney, B. W., Laird, J. B., \& Latham, D. W. 1988, AJ, 96, 560

Carretta, E., Gratton, R. G., \& Clemintini, G. 2000, MNRAS, 316, 721

Carretta, E., Gratton, R. G., Clementini, G., \& Pecci, F. F. 2000, ApJ, 533, 215

Chen, L., Geffert, M., Wang, J. J., Reif, K., \& Braun, J. M. 2000, A\&AS, 145, 223

Chiba, M., \& Beers, T. C. 2001, ApJ, 549, 325

Christlieb, N., Wisotzky, L., Reimers, D., et al. 2001, A\&A, 366, 898

Côté, P., Mateo, M., Sargent, W. L. W., \& Olszewski, W. 2000, ApJ, 537, L91

Dauphole, B., \& Colin, J. 1995, A\&A, 300, 117

${ }^{3}$ For example, if $10 \%$ of our FHB sample is contaminated by blue metal-poor stars, we obtain a $2 \sim 3 \times 10^{11} M_{\odot}$ decrease in our total mass estimate, based on Monte Carlo experiments. 
Dehnen, W., \& Binney, J. J. 1998, MNRAS, 298, 387

Dejonghe, H. 1986, Phys. Rep., 133, 217

Dinescu, D. I., Girard, T. M., \& van Altena, W. F. 1999, AJ, 117, 1792

Dinescu, D. I., Majewski, S. R., Girard, T. M., \& Cudworth, K. M. 2000, AJ, 120, 1892

Dinescu, D. I., Majewski, S. R., Girard, T. M., \& Cudworth, K. M. 2001, AJ, 122, 1916

Evans, N. W., \& Wilkinson, M. I. 2000, MNRAS, 316, 929

Evans, N. W., Wilkinson, M. I., Guhathakurta, P., Grebel, E. K., \& Vogt, S. S. 2000, ApJ, 540, L9

Fich, M., \& Tremaine, S. 1991, ARA\&A, 29, 409

Fricke, V. W. 1949, Astr. Nachr., 278, 49

Harris, W. E. 1996, AJ, 112, 1487

Held, E. V., Clementini, G., Rizzi, L., et al. 2001, ApJ, 562, L39

Hernquist, L. 1990, ApJ, 356, 359

Hog, E., Fabricius, C., Makarov, V. V., et al. 2000, A\&A, 355, L27

Honma, M., \& Sofue, Y. 1997, PASJ, 49, 453

Honma, M., \& Kan-ya, Y. 1998, ApJ, 503, L139

Honma, M., Kawaguchi, N., \& Sasao 2000, in Proc. SPIE 4015, Radio Telescope, ed. H. R. Butcher, 624

Ibata, R., Lewis, G. F., Irwin, M., Totten, E., \& Quinn, T. 2001, ApJ, 551,294

Irwin, M., Ibata, R., Gilmore, G., Wyse, R., \& Suntzeff, N. 1996, in Formation of the Galactic Halo - Inside and Out, ed. H. Morrison, \& A. Sarajedini (San Francisco: ASP), ASP Conf. Ser., 92, 841

Johnston, K. V., Spergel, D. N., \& Hernquist, L. 1995, ApJ, 451, 598

Johnston, K. V., Zhao, H., Spergel, D. N., \& Hernquist, L. 1999, ApJ, 512, L109

Kerr, F. J., \& Lynden-Bell, D. 1986, MNRAS, 221, 1023

Kinman, T. D., Suntzeff, N. B., \& Kraft, R. P. 1994, AJ, 108, 1722

Klemola, A. R., Hanson, R. B., Jones, B. F. 1994, Lick Northern Proper Motion Program: NPM1 Catalog (NSSDC/ADC Cat. A1199) (Greenbelt, MD: GSFC)

Kochanek, C. S. 1996, ApJ, 457, 228

Kroupa, P., \& Bastian, U. 1997, New Astron., 2, 77

Leonard, P. J. T., \& Tremaine, S. 1990, ApJ, 353, 486

Lindegren, L., \& Perryman, M. A. C. 1996, A\&A, 116, 579

Little, B., \& Tremaine, S. 1987, ApJ, 320, 493

Martin, J. C., \& Morrison, H. L. 1998, AJ, 116, 1724

Mateo, M. 1998, ARA\&A, 36, 435

Méndez, R. A., Platais, I., Girard, T. M., Kozhurina-Platatis, V., \& van Altena, W. F. 1999, ApJ, 524, L39

Merritt, D., \& Tremblay, B. 1993, AJ, 106, 2229
Mihalas, D. \& Binney, J. 1981, Galactic Astronomy: Structure and Kinematics, 2nd ed. (Freeman, San Francisco)

Miyamoto, M., \& Nagai, R. 1975, PASJ, 27, 533

Miyamoto, M., \& Zhu, Z. 1998, AJ, 115, 1483

Miyamoto, M., Satoh, C., \& Ohashi, M. 1980, A\&A, 90, 215

Norris, J. E., \& Hawkins, M. R. S. 1991, ApJ, 380, 104

Peebles, P. J. E. 1995, ApJ, 449, 52

Pedreros, M. H., Anguita, C., \& Maza, J. 2002, AJ, 123, 1971

Platais, I., Girard, T. M., Kozhurina-Platais, V., et al. 1998, AJ, 116, 2556

Press, W. H., Teukolsky, S. A., Vetterling, W. T., \& Flannery, B. P. 1992, Numerical Recipes in Fortran 77: the art of scientific computing, 2nd ed. (Cambridge University Press, Cambridge)

Preston, G. W., \& Sneden, C. 2000, AJ, 120, 1014

Preston, G. W., Beers, T. C., \& Shectman, S. A. 1994, AJ, 108, 538

Preston, G. W., Shectman, S. A., \& Beers, T. C. 1991, ApJ, 375, 121

Rodgers, A. W., \& Roberts, W. H. 1993, AJ, 106, 1839

Röser, S. 1996, in IAU Symp. 172, ed. S. Ferraz-Mello et al. (Dordrecht: Kluwer), 481

Sasao, T. 1996, in Proc. 4th Asia-Pacific Telescope Workshop, ed. E. A. King (Sidney: Australian Telescope National Facility), 94

Schmoldt, I. M., \& Saha, P. 1998, AJ, 115, 2231

Scholz, R.-D., \& Irwin, M. J. 1994, in Astronomy from Wide-Field Imaging, ed. MacGillivray, H. T., Thomson, E. B., Lasker, et al. (Dordrecht: Kluwer), IAU Symp., 161, 535

Siegel, M. H., Majewski, S. R., Cudworth, K. M., \& Takamiya, M. 2001, AJ, 121, 935

Sommer-Larsen, J., Beers, T. C., Flynn, C., Wilhelm, R., \& Christensen, P. R. 1997, ApJ, 481, 775

Unwin, S., Boden, A., \& Shao, M. 1997, Proc. STAIF, AIP Conf. Proc., 387, 63

Wang, J. J., Chen, L. L., Wu, Z. Y., Gupta, A. C., \& Geffert, M. 2000, A\&AS, 142, 373

Wilkinson, M. I., \& Evans, N. W. 1999, MNRAS, 310, 645 (WE99)

Wilhelm, R., Beers, T. C., \& Gray, R. O. 1999a, AJ, 117, 2308

Wilhelm, R., Beers, T. C., Sommer-Larsen, J., et al. 1999b, AJ, 117, 2329

Zacharias, N., Urban, S. E., Zacharias, M. I., et al. 2000, AJ, 120, 2131

Zacharias, N., Zacharias, M. I., Urban, S. E., \& Rafferty, T. J. 2001, BAAS, 199, 129.08

Zaritsky, D., Olszewski, E. W., Schommer, R. A., Peterson, R. C., \& Aaronson, M. 1989, ApJ, 345, 759 